\title{
Real-time navigation by three-dimensional virtual reconstruction models in robot-assisted laparoscopic pyeloplasty for ureteropelvic junction obstruction: our initial experience
}

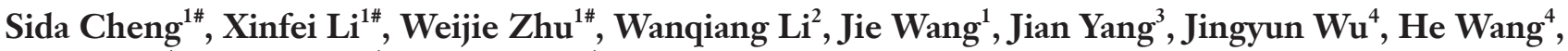 \\ Lei Zhang ${ }^{1}$, Xuesong Li $^{1}$, Liqun Zhou ${ }^{1}$
}

${ }^{1}$ Department of Urology, Peking University First Hospital, Institute of Urology, Peking University, National Urological Cancer Center, Beijing, China; ${ }^{2}$ Department of Urology, The First College of Clinical Medical Science, Three Gorges University/Yichang Central People's Hospital, Yichang, China; ${ }^{3}$ Laboratory of Beijing Engineering Research Center of Mixed Reality and Advanced Display, School of Optics and Photonics, Beijing Institute of Technology, Beijing, China; ${ }^{4}$ Department of Medical Imaging, Peking University First Hospital, Beijing, China

Contributions: (I) Conception and design: L Zhang, X Li; (II) Administrative support: X Li; (III) Provision of study materials or patients: S Cheng, X Li; (IV) Collection and assembly of data: S Cheng, X Li, W Zhu; (V) Data analysis and interpretation: S Cheng, X Li, W Zhu; (VI) Manuscript writing: All authors; (VII) Final approval of manuscript: All authors.

\#These authors contributed equally to this work.

Correspondence to: Lei Zhang; Xuesong Li. Department of Urology, Peking University First Hospital, Institute of Urology, Peking University, National Urological Cancer Center, Beijing 100034, China. Email: zl070501@126.com; pineneedle@sina.com.

Background: The aim of this study was to evaluate the effectiveness and safety of real-time surgical navigation by three-dimensional (3D) virtual reconstruction models in robot-assisted laparoscopic pyeloplasty (RALP).

Methods: Between November 2018 and January 2020, 38 patients with ureteropelvic junction obstruction (UPJO) who underwent RALP were retrospectively enrolled. The operations were assisted in real time by $3 \mathrm{D}$ models in 16 patients, while 22 patients underwent surgery without navigation. Based on whether patients had a prior intervention history, crossing vessels or congenital deformities, we further divided them into the "complicated UPJO" cohort and the "regular UPJO" cohort for subgroup analysis. The demographic characteristics, intraoperative parameters, perioperative data and follow-up data were recorded and compared between the groups.

Results: All of the procedures were successfully performed without open or laparoscopic conversion. The mean dissection time to the UPJ was shorter in the navigation group than in the non-navigation group, both in the whole cohort (15.3 vs. $24.8 \mathrm{~min}, \mathrm{P}=0.011$ ) and in the complicated cohort (15.4 vs. $27.5 \mathrm{~min}, \mathrm{P}=0.004)$, while there was no significant difference in the regular cohort. The overall operative time and estimated blood loss in the navigation group tended to be less, although the difference was not statistically significant. No difference in anastomosis time, postoperative hospital stay or complications was noted between the two groups in either cohort. At a mean follow-up of 11.2 months, the overall success rate was $94.7 \%$ (36/38), and there was no significant difference between the two groups.

Conclusions: Real-time navigation by $3 \mathrm{D}$ virtual reconstruction models might be helpful to improve surgical efficiency and safety of RALP by facilitating the dissection around the UPJ, especially for cases of complicated UPJO. However, the prospective study with larger sample size is further needed to confirm the results.

Keywords: Three-dimensional reconstruction model; surgical navigation; robot-assisted laparoscopic pyeloplasty (RALP); ureteropelvic junction dissection

Submitted Jun 16, 2020. Accepted for publication Oct 16, 2020.

doi: $10.21037 /$ tau-20-1006

View this article at: http://dx.doi.org/10.21037/tau-20-1006 


\section{Introduction}

Pyeloplasty has been the gold standard surgical management of ureteropelvic junction obstruction (UPJO) since its introduction (1). In recent years, laparoscopic pyeloplasty (LP) and robot-assisted laparoscopic pyeloplasty (RALP) have become first-line options with high success rates, better cosmetic results, faster recovery and less pain $(2,3)$. In particular, the unique advantages of robotic platforms, including three-dimensional (3D) visualization and multidirectional EndoWrist systems, have greatly facilitated the intracorporeal cutting and suturing to reduce the operation difficulty $(2,4)$.

In these cases, the most challenging surgical step might be converted to the dissection around the ureteropelvic junction (UPJ) rather than intracorporeal anastomosis in $\operatorname{RALP}(5,6)$. The surrounding abnormal anatomy makes it technically challenging even for experienced surgeons to identify and dissect the UPJ, especially in complicated scenarios, such as recurrent UPJO with dense fibrosis scars, UPJO with concomitant congenital malformations and UPJO with crossing vessels (7-9). Although the robotic platforms have provided great advantages for pyeloplasty, there remain unmet needs for the application of navigation tools to locate the UPJ and further improve surgical efficiency and safety.

Rapid developments in imaging technology, such as 3D virtual reconstruction, $3 \mathrm{D}$ printing, and augmented reality (AR), have provided new options for personalized surgical management (10). The application of $3 \mathrm{D}$ reconstruction in urology has been reported in various fields with satisfactory results, such as in calculus disease, renal neoplasms and prostate cancer (11-13). In our center, the $3 \mathrm{D}$ reconstruction has also been used for the management of ureteral strictures, including diagnosis, patient education, surgical planning, intraoperative navigation and outcomes measurement. However, few published studies have focused on this special field, and no study has evaluated its efficiency as a surgical navigation tool in pyeloplasty by comparative analysis.

To further improve the surgical efficiency and safety of pyeloplasty, we reviewed our experience using the $3 \mathrm{D}$ virtual reconstruction model as a real-time surgical navigation tool in RALP and evaluated its safety, feasibility and effectiveness by comparing perioperative parameters for the first time. We present the following article in accordance with the STROBE reporting checklist (available at http://dx.doi.org/10.21037/tau-20-1006).

\section{Methods}

\section{Study population}

We retrospectively reviewed 38 patients who underwent RALP for UPJO in our center between November 2018 and January 2020. RALP was performed in 16 patients with real-time navigation by $3 \mathrm{D}$ models, while the other 22 patients underwent RALP without navigation. All of the patients were treated in the same period by a single experienced surgeon. Patient demographics, perioperative variables, postoperative complications and follow-up data were recorded and compared between the navigation and non-navigation groups.

All patients were preoperatively diagnosed with UPJO based on radiographic examinations, such as diuretic renography, computerized tomography (CT), and intravenous or retrograde pyelography. Indications for surgery included recurrent flank pain, recurrent pyelonephritis, evident anatomic obstruction based on images and deterioration of renal function. The standardized dismembered pyeloplasty technique was used as we previously reported (14), and all surgeries were performed in a four-arm transperitoneal manner by the da Vinci Si robot platform (Intuitive Surgical, Sunnyvale, CA, USA). Patients were excluded from the analysis if the peri- or postoperative data were missing. All procedures performed in this study were in accordance with the Declaration of Helsinki (as revised in 2013) and approved by the Ethics Committee of Peking University First Hospital (approval number: 2020-283). Because of the retrospective nature of the research, the requirement for informed consent was waived.

\section{Measurements and outcomes}

The efficiency of the 3D model navigation was evaluated by operative times, recorded as the overall operative time (from skin incision until wound closure, excluding calculus removal), dissection time to UPJ (from the opening of Gerota's fascia until the exposure completion immediately before pelvic incision) and anastomosis time (from the first stitch to the completion of pyeloplasty anastomosis). Safety was evaluated by the estimated blood loss and complications (according to the Clavien-Dindo classification system) (15). For further analysis, we defined secondary UPJO, the appearance of crossing vessels in the dissection area, and concomitant horseshoe kidney as the "complicated UPJO" cohort in the current study. The patients not meeting any the above criteria were categorized into the "regular UJPO" cohort.

Postoperative follow-up was performed at 3 and 

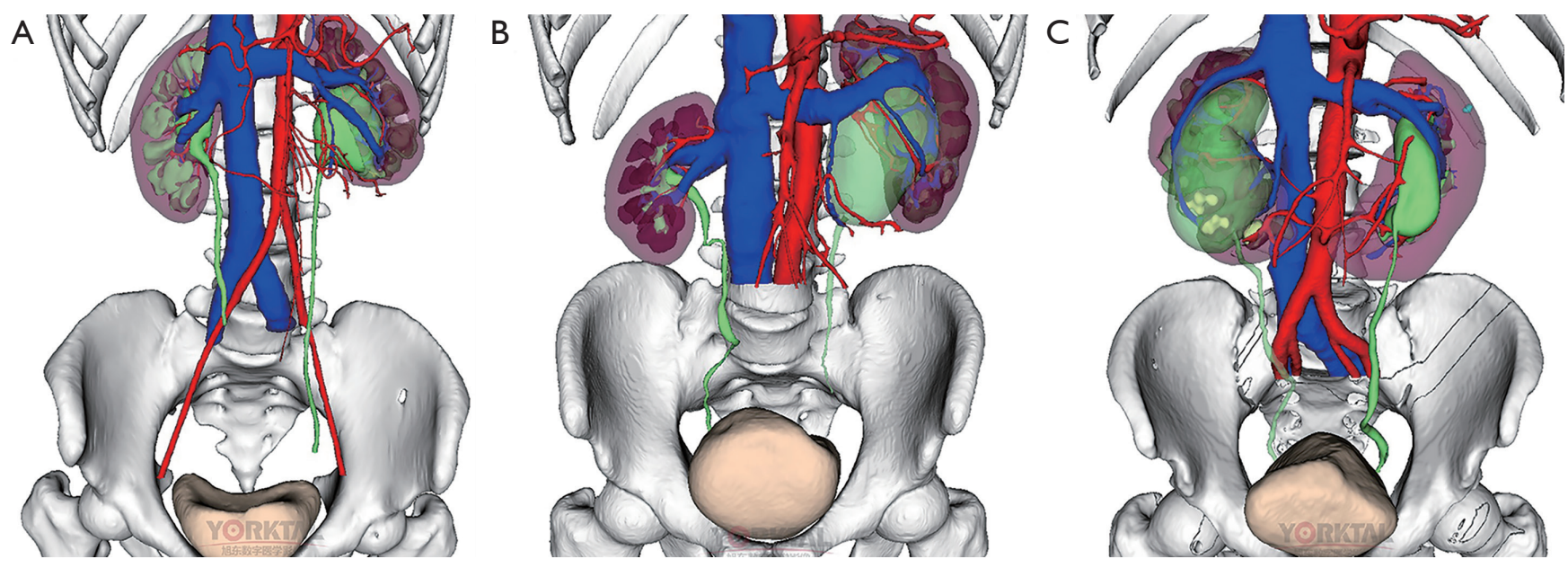

Figure 1 The 3D virtual reconstruction models. The models include semitransparent kidney (in reddish brown), collecting system (in green), blood vessels (arteries in red and veins in blue) and potential calculus (in yellow). (A) The 3D model of one case with tangled crossing vessels. (B) The 3D model of one case with failed prior intervention history. (C) The 3D model of one case with horseshoe kidney. 3D, three-dimensional.

6 months and then annually. The follow-up examinations included ultrasonography, retrograde pyelography, renography and CT. Success criteria were the alleviation of subjective pain, the improvement of hydronephrosis and the stable or improved renal function.

\section{$3 D$ virtual reconstruction and surgical navigation}

Preoperative enhanced CT images were acquired by a 64 multidetector-row CT scanner (Philips, Best, The Netherlands) with $0.5-\mathrm{mm}$ step intervals. The data used for the subsequent analysis were stored in the Digital Imaging and Communications in Medicine (DICOM) format. We used independently developed reconstruction model processing software, IPS (Yorktal, Inc., Shenzhen, Guangdong, China) to perform the 3D reconstruction. By manual adjustments, 2D cross-sectional CT imaging in 3.0 DICOM format could be segmented and reconstructed semiautomatically into a $3 \mathrm{D}$ virtual reconstruction model using this software. The $3 \mathrm{D}$ surface-rendered semitransparent renal parenchyma, collecting system, local blood vessels, and potential calculus were included in the formed models with different colors (Figure 1). Each section of the reconstructed model allows for appearance/ disappearance, zooming in/out and rotation at any angle.

During surgery, the $3 \mathrm{D}$ model was imported inside the robotic console and displayed alongside the surgical field. According to the real-time surgical field images, surgeons can control the appearance/disappearance of the model, while assistants can rotate and zoom it synchronously to match the anatomical landmarks. Based on the 3D models, the surgeons can have a comprehensive understanding of the anatomic structures in operative region. Under its guidance, the peri-UPJ dissection can be conducted in the proper region and in a targeted spatial direction. It can help surgeons access the UPJ in the shortest distance and avoid the underlying injuries (Figure 2).

\section{Statistical analysis}

Statistical analysis was performed using SPSS 25.0 (IBM Corporation, Armonk, NY, USA). Categorical variables were presented as frequencies and percentages. Continuous parametric variables were shown as the mean \pm standard deviation and nonparametric variables were expressed as median (interquartile range). The Fisher's exact test was used for the comparison between categorical variables, while the Student $t$-test or Mann-Whitney $\mathrm{U}$ test was performed for continuous variables. A two-sided $\mathrm{P}<0.05$ defined statistical significance.

\section{Results}

\section{Clinical characteristics of patients}

A total of 38 patients, consisting of 21 males and 17 females, 


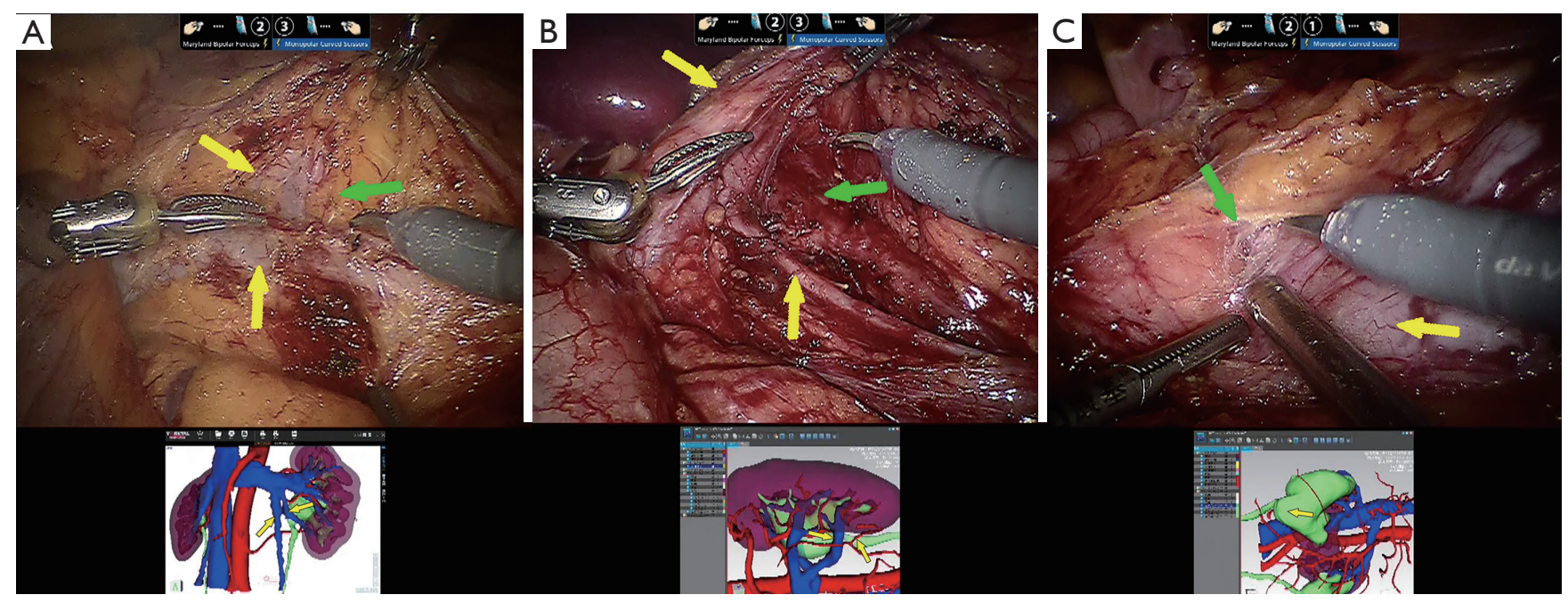

Figure 2 Intraoperative real-time navigation by the 3D models in RALP. (A) The navigation in one case with anterior crossing vessels. Two anterior venae were indicated by yellow arrows both in the $3 \mathrm{D}$ model and the surgical field. The green arrow pointed out the proper dissection area of the next step. The surgeon can access the UPJ directly without encountering any other structure as shown in 3D model. (B) The navigation in a recurrent UJPO case. One anterior vena and one arteriole were indicated by yellow arrows both in the 3D model and the surgical field. The green arrow pointed out the proper dissection area of the next step. (C) The navigation in one case with horseshoe kidney. The lowest pole of abnormal pelvic was indicated by the yellow arrow and the potential location of UPJ was pointed out by the green arrow. The quick localization of UPJ and confirmation of the absence of aberrant vessels greatly simplified the dissection step. 3D, threedimensional; RALP, robot-assisted laparoscopic pyeloplasty; UPJ, ureteropelvic junction; UJPO, ureteropelvic junction obstruction.

were enrolled in this study. The mean age of the patients was 32.5 years old. Twenty-one $(55.3 \%)$ patients complained of lateral flank pain, $3(7.9 \%)$ patients presented with hematuria, and $16(42.1 \%)$ patients were asymptomatic. All patients presented with moderate or severe hydronephrosis and were diagnosed with UPJO, with 18 left-sided, 15 right-sided and 5 bilateral cases. Six patients had prior interventions for UPJO. Fifteen patients had crossing vessels in the dissection area and 5 patients had horseshoe kidneys (Table 1).

\section{Comparison between the navigation and the non- navigation groups}

Sixteen patients underwent RALP with the real-time navigation by $3 \mathrm{D}$ models, while the other 22 patients underwent regular RALP. All procedures were successfully performed without open or laparoscopic conversion. There were no significant differences between the two groups in terms of age, BMI (body mass index), laterality, previous intervention history, malformation or crossing vessels. We found that the mean dissection time to the UPJ in the navigation group was shorter than that in the non-navigation group (15.3 vs. $24.8 \mathrm{~min}, \mathrm{P}=0.011$ ). There were no differences in overall operative time, anastomosis time, estimated blood loss, postoperative hospital stay or complications between the two groups. One major postoperative complication (grade IIIa) occurred in the nonnavigation group. This patient had urinary extravasation postoperatively due to a misplaced DJ stent, which required a second surgery for stent replacement (Table 2).

We further compared the intraoperative parameters and perioperative outcomes in the "complicated UPJO" and the "regular UPJO" cohorts. In the complicated UPJO cohort, we found that the mean dissection time to the UPJ was significantly shorter in the navigation group (15.4 vs. $27.5 \mathrm{~min}, \mathrm{P}=0.004)$. Meanwhile, there were trends towards a reduced overall operative time and estimated blood loss in the navigation group of the complicated cohort, although the difference was not statistically significant (Table 3). Conversely, there was no significant difference in the dissection time between the two groups in the regular cohort (14.6 vs. $21.6 \mathrm{~min}, \mathrm{P}=0.457$ ) (data not shown).

\section{Follow-up data}

All 38 patients were followed up successfully. During the 
Table 1 Demographic and clinical data of all patients

\begin{tabular}{|c|c|}
\hline Variable & Value \\
\hline Number of patients, $n$ & 38 \\
\hline \multicolumn{2}{|l|}{ Gender, n } \\
\hline Male & 21 \\
\hline Female & 17 \\
\hline Age, years, mean $\pm S D$ & $32.5 \pm 11.9$ \\
\hline $\mathrm{BMI}, \mathrm{kg} / \mathrm{m}^{2}$, mean $\pm \mathrm{SD}$ & $23.0 \pm 4.1$ \\
\hline \multicolumn{2}{|l|}{ Chief complaints, $n$} \\
\hline Flank pain & 21 \\
\hline Hematuria & 3 \\
\hline Asymptomatic & 16 \\
\hline \multicolumn{2}{|l|}{ Affected side, $n$} \\
\hline Left & 18 \\
\hline Right & 15 \\
\hline Bilateral & 5 \\
\hline \multicolumn{2}{|l|}{ Concurrent complications, $\mathrm{n}$} \\
\hline Crossing vessels & 15 \\
\hline Calculi & 14 \\
\hline Horseshoe kidney & 5 \\
\hline \multicolumn{2}{|l|}{ Previous interventions for UPJO, $\mathrm{n}$} \\
\hline Laparoscopic pyeloplasty & 2 \\
\hline Balloon dilation & 1 \\
\hline Ureteroureterostomy & 2 \\
\hline \multicolumn{2}{|l|}{ Multiple interventions } \\
\hline $\begin{array}{l}\text { Laparoscopic pyeloplasty plus balloon } \\
\text { dilation }\end{array}$ & 1 \\
\hline
\end{tabular}

BMI, body mass index; UPJO, ureteropelvic junction obstruction.

mean follow-up of 11.2 months, all of the patients had stable or improved renal function and patent drainage without obstruction. All patients with preoperative flank pain experienced improvement, among whom 12 patients (57.1\%) experienced complete pain resolution. Thirtysix patients $(94.7 \%)$ who demonstrated no or mild hydronephrosis were regarded as having hydronephrosis improvement. Two patients in the non-navigation group experienced persistent hydronephrosis, but they remained asymptomatic and their renal function was stable. The overall success rate was $94.7 \%(36 / 38)$, and there was no significant difference between the two groups (100\% vs. $90.9 \%, \mathrm{P}=0.499)$.

\section{Discussion}

Anderson-Hynes dismembered pyeloplasty, which can resect the stenotic or adynamic segment and reduce the enlarged renal pelvis, can be applied in various scenarios (16). It has exhibited a consistently high success rate since its introduction (1). Following the development of better instruments and skilled intracorporeal techniques, laparoscopic and robot-assisted dismembered pyeloplasty has demonstrated promising success rates and been widely adopted because of the better cosmetic results, shorter recovery time and reduced morbidity (2).

Currently, the most challenging surgical step of pyeloplasty is no longer intracorporeal tailoring or suturing because of the unique features provided by robotic platforms, including $3 \mathrm{D}$ visualization, reduced tremor interference and improved dexterity of the articulated EndoWrist $(2,17)$. In contrast, surgeons might encounter the most individualized variation during exposure of the UPJ (5). The changeable structures cause many urologists to be instinctively cautious and easily lost, leading to increased mental and physical fatigue, and a higher risk of tissue injury. Receiving more accurate information on the UPJ location and aberrant anatomic structures where potential surgical injury is likely to occur is critical for UPJ dissection.

Surgeons' current understanding of peri-UPJ anatomy is mainly based on preoperative CT images along with intraoperative visualization. It is probably sufficient for surgeons to create a mental image of the UPJ location and relevant vessels in simple scenarios. However, this approach becomes less effective for cases of complicated UPJO with abnormal anatomy, which cannot be displayed intuitively on $2 \mathrm{D}$ cross-sectional images. Therefore, the development of new navigation tools is indispensable to reduce surgical difficulty and improve surgical safety and efficiency.

Advancements in image technology have allowed surgeons to get detailed and case-specific perceptions of the surgical anatomy, facilitating surgical planning, realtime guidance, skill training and patient education (10). 3D printing models can be handled easily and have gained increasing popularity in recent years (18). However, the additional cost and preparation time have limited its widespread application (19). AR can greatly enhance intraoperative understanding by overlaying $3 \mathrm{D}$ images on 
Table 2 Clinical characteristics, perioperative data and follow-up data of the navigation and non-navigation groups

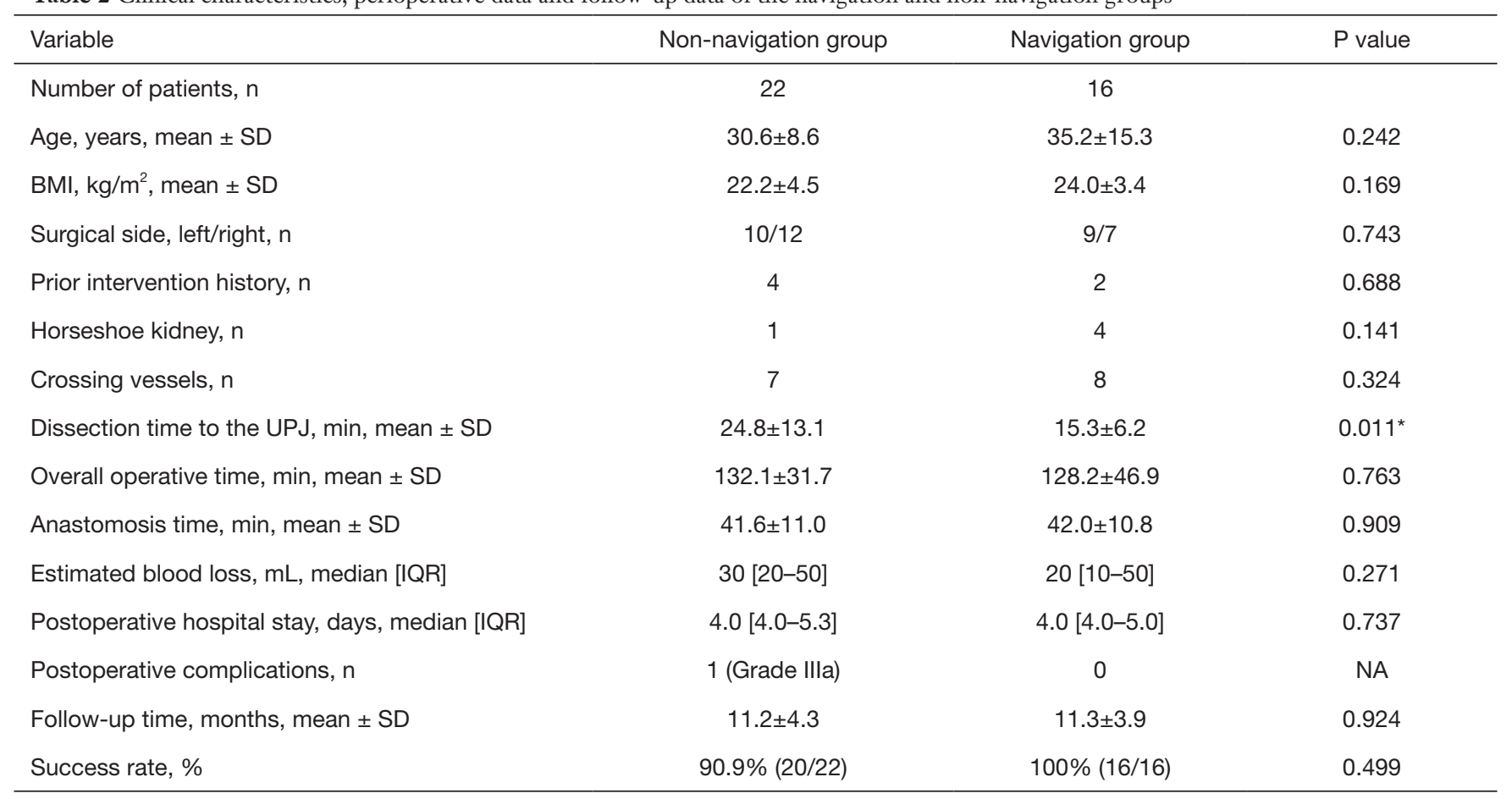

*, P<0.05. BMI, body mass index; UPJ, ureteropelvic junction; SD, standard deviation; IQR, interquartile range; NA, not available.

Table 3 Clinical characteristics, perioperative data and follow-up data in the complicated UPJO cohort

\begin{tabular}{|c|c|c|c|}
\hline Variable & Non-navigation group & Navigation group & $P$ value \\
\hline Age, years, mean \pm SD & $30.4 \pm 10.4$ & $38.7 \pm 14.8$ & 0.123 \\
\hline $\mathrm{BMI}, \mathrm{kg} / \mathrm{m}^{2}$, mean $\pm \mathrm{SD}$ & $22.6 \pm 5.0$ & $23.6 \pm 3.1$ & 0.542 \\
\hline Surgical side, left/right, $n$ & $7 / 5$ & $6 / 7$ & 0.695 \\
\hline Horseshoe kidney, $\mathrm{n}$ & 1 & 4 & 0.322 \\
\hline Crossing vessels, $n$ & 7 & 8 & 1.000 \\
\hline Dissection time to the UPJ, min, mean \pm SD & $27.5 \pm 11.8$ & $15.4 \pm 5.8$ & $0.004^{*}$ \\
\hline Overall operative time, min, mean \pm SD & $137.6 \pm 30.0$ & $123.7 \pm 42.2$ & 0.353 \\
\hline Postoperative hospital stay, days, median [IQR] & $4.0[4.0-5.8]$ & $4.0[4.0-5.0]$ & 0.650 \\
\hline Postoperative complications, $n$ & 1 (Grade IIla) & 0 & NA \\
\hline Follow-up time, months, mean $\pm S D$ & $11.7 \pm 4.5$ & $11.8 \pm 3.7$ & 0.951 \\
\hline Success rate, \% & $83.3 \%(10 / 12)$ & $100 \%(13 / 13)$ & 0.220 \\
\hline
\end{tabular}

*, P<0.05. UPJO, ureteropelvic junction obstruction; BMI, body mass index; UPJ, ureteropelvic junction; SD, standard deviation; IQR, interquartile range; NA, not available. 
the operative field. However, the automatic registration is immature and manual superimposition is still required in most cases, along with the requirement of complicated devices, making it far from being available for daily use (10). In contrast, 3D virtual reconstruction models obtained from CT or MRI are relatively inexpensive and easily acquired without obvious technical barriers, so they can be highly accessible for most centers.

In urologic surgery, the $3 \mathrm{D}$ reconstruction has been mainly implemented in the management of prostate and kidney cancer $(11,20,21)$, while quite limited exploration in the urinary reconstruction area has been reported. He et al. described that CT angiography with $3 \mathrm{D}$ reconstruction was reliable in the diagnosis of hydronephrosis caused by aberrant renal vessels (22). Similarly, Wang et al. reported that the $3 \mathrm{D}-\mathrm{MR}$ angiography had a high prediction rate of $83 \%$ for the crossing vessels (23). As for surgical simulation, Cheung et al. reported that the $3 \mathrm{D}$-printed model is a promising tool in laparoscopic pyeloplasty, while Bendre et al. demonstrated its role in robotic pyeloplasty $(24,25)$. However, there are still no available reports to date of the real-time intraoperative navigation by $3 \mathrm{D}$ reconstruction models in UPJO treatment.

For the first time, we used quantified methods to investigate the efficiency of $3 \mathrm{D}$ virtual reconstruction models for surgical navigation in pyeloplasty. We found that 3D model navigation could promote the efficiency of one critical step: exposure of the UPJ. The dissection to the UPJ in the navigation group took approximately one-third less of the time that it took in the non-navigation group $(\mathrm{P}=0.011)$. Although dissecting the ureter and renal pelvis only accounts for a small proportion of the whole procedure, it might be the most challenging step in the robotic era and the step at which there remains room for improvement. Under the navigation of $3 \mathrm{D}$ models, the surgeon can clearly understand the UPJ location and predict potential anatomic structures in the perirenal fat tissue or fibrosis scar. Once intact exposure of the dilated pelvis and UPJO is accomplished, the subsequent cutting and anastomosis steps can be regarded as standardized procedures without much variation (5).

The messy anatomical structures in some complicated situations are much more difficult to manage, even for experienced surgeons. Baek (5) found that the exposure time of the UPJ was $86.4 \%$ longer for reperformed cases than primary cases and blamed it on scar formation after the previous intervention. The scar formation and neovascularization in secondary UPJO might lead to an extended operative time and increased estimated blood loss, as confirmed by other studies (8,26-28). Besides, the concomitant crossing vessels can lead to complications if incautious isolation is conducted and vessel injury occurs (16). Although the percentage of vessel injury is quite low, less than $2.5 \%$ according to a previous report (29), careful identification and meticulous dissection of the vessel are time consuming and raise high technical requirements (7). In addition, congenital malformations, such as horseshoe kidneys, have quite different anatomic structures and adjacency relationships. They are also prone to have crossing vessels due to the anomalous blood supply (30).

By subgroup analysis, we found that 3D model navigation might be more helpful in complicated situations, saving almost half the time of UPJ dissection ( $\mathrm{P}=0.004)$. The more accurate and real-time intraoperative information might be the reason for the favorable outcomes. Interestingly, no obvious improvement of the dissection step was observed in the regular cohort. One likely explanation was that all of the surgeries were performed by an experienced surgeon who has performed more than 200 laparoscopic or robot-assisted pyeloplasty procedures. We believe that there is no need for experienced surgeons to use $3 \mathrm{D}$ navigation in simple cases. However, it might still be helpful for beginners because of the complexity and relatively steep learning curve of pyeloplasty.

Furthermore, no intraoperative complication occurred and there was no difference in the estimated blood loss or postoperative complications between the navigation and non-navigation groups, indicating that the $3 \mathrm{D}$ models can be a safe tool for surgical navigation. Besides, the navigation group showed a trend towards a less estimated blood loss, although the difference was not statistically significant, which might be partially due to the relatively small sample size. A future larger cohort is needed to confirm the protective effect of $3 \mathrm{D}$ navigation on vessel or tissue injuries. Notably, the success rates were $90.9 \%$ and $100 \%$ in the navigation and non-navigation groups, respectively, which were comparable or even better than those in previous RALP reports $(3,6)$. These results demonstrate that RALP is feasible and effective and that the greatest benefit of 3D navigation might lie in improving the surgical efficiency and safety, especially for complicated UPJO.

There are several limitations of our study. It was a retrospective and single-center study, which might have resulted in selection bias. Additionally, the sample size of this study was small, which might affect the stability of our findings. Therefore, a prospective and randomized study 
with a larger sample size is required to further validate our results. Moreover, all of the surgeries in our study were performed by an experienced surgeon, and further evaluation should also be conducted in inexperienced beginners.

\section{Conclusions}

Based on these initial results, we presume that the observed improvement of dissection step was probably resulted from the application of real-time navigation by $3 \mathrm{D}$ virtual reconstruction models. It might be helpful to reduce the difficulty of this critical step, dissection around the UPJ, and improve surgical efficiency and safety, especially for complicated cases of UPJO in RALP. However, a prospective and randomized study with larger sample size is warranted to better delineate the role of this navigation tool in the treatment of UPJO.

\section{Acknowledgments}

The authors thank the entire staff of the Department of Urology, Peking University First Hospital.

Funding: None.

\section{Footnote}

Reporting Checklist: The authors have completed the STROBE reporting checklist. Available at http://dx.doi. org/10.21037/tau-20-1006

Data Sharing Statement: Available at http://dx.doi. org/10.21037/tau-20-1006

Conflicts of Interest: All authors have completed the ICMJE uniform disclosure form (available at http://dx.doi. org/10.21037/tau-20-1006). Dr. Xuesong L serves as an unpaid editorial board member of Translational Andrology and Urology. Dr. Liqun $Z$ serves as an unpaid editorial board member of Translational Andrology and Urology from Mar 2012 to Feb 2020. The other authors have no conflicts of interest to declare.

Ethical Statement: The authors are accountable for all aspects of the work in ensuring that questions related to the accuracy or integrity of any part of the work are appropriately investigated and resolved. All procedures performed in this study were in accordance with the
Declaration of Helsinki (as revised in 2013) and approved by the Ethics Committee of Peking University First Hospital (approval number: 2020-283). Because of the retrospective nature of the research, the requirement for informed consent was waived.

Open Access Statement: This is an Open Access article distributed in accordance with the Creative Commons Attribution-NonCommercial-NoDerivs 4.0 International License (CC BY-NC-ND 4.0), which permits the noncommercial replication and distribution of the article with the strict proviso that no changes or edits are made and the original work is properly cited (including links to both the formal publication through the relevant DOI and the license). See: https://creativecommons.org/licenses/by-nc-nd/4.0/.

\section{References}

1. O'Reilly PH, Brooman PJ, Mak S, et al. The longterm results of Anderson-Hynes pyeloplasty. BJU Int 2001;87:287-9.

2. Autorino R, Eden C, El-Ghoneimi A, et al. Robotassisted and laparoscopic repair of ureteropelvic junction obstruction: a systematic review and meta-analysis. Eur Urol 2014;65:430-52.

3. Gupta NP, Nayyar R, Hemal AK, et al. Outcome analysis of robotic pyeloplasty: a large single-centre experience. BJU Int 2010;105:980-3.

4. Yohannes P, Burjonrappa SC. Rapid communication: laparoscopic Anderson-Hynes dismembered pyeloplasty using the da Vinci robot: technical considerations. J Endourol 2003;17:79-83.

5. Baek M, Silay MS, Au JK, et al. Quantifying the Additional Difficulty of Pediatric Robot-Assisted Laparoscopic Re-Do Pyeloplasty: A Comparison of Primary and Re-Do Procedures. J Laparoendosc Adv Surg Tech A 2018;28:610-6.

6. Niver BE, Agalliu I, Bareket R, et al. Analysis of roboticassisted laparoscopic pyleloplasty for primary versus secondary repair in 119 consecutive cases. Urology 2012;79:689-94.

7. Shoma AM, El Nahas AR, Bazeed MA. Laparoscopic pyeloplasty: a prospective randomized comparison between the transperitoneal approach and retroperitoneoscopy. J Urol 2007;178:2020-4; discussion 2024.

8. Dirie NI, Ahmed MA, Wang S. Is secondary robotic pyeloplasty safe and effective as primary robotic pyeloplasty? A systematic review and meta-analysis. J 
Robot Surg 2020;14:241-8.

9. Parma P, Samuelli A, Luciano M, et al. Salvage laparoscopic pyeloplasty in the worst case scenario: after failed open repair and endoscopic salvage. Urologia 2014;81 Suppl 23:S9-14.

10. Porpiglia F, Amparore D, Checcucci E, et al. Current Use of Three-dimensional Model Technology in Urology: A Road Map for Personalised Surgical Planning. Eur Urol Focus 2018;4:652-6.

11. Ukimura O, Nakamoto M, Gill IS. Three-dimensional reconstruction of renovascular-tumor anatomy to facilitate zero-ischemia partial nephrectomy. Eur Urol 2012;61:211-7.

12. Li H, Chen Y, Liu C, et al. Construction of a threedimensional model of renal stones: comprehensive planning for percutaneous nephrolithotomy and assistance in surgery. World J Urol 2013;31:1587-92.

13. Porpiglia F, Checcucci E, Amparore D, et al. Threedimensional Elastic Augmented-reality Robot-assisted Radical Prostatectomy Using Hyperaccuracy Threedimensional Reconstruction Technology: A Step Further in the Identification of Capsular Involvement. Eur Urol 2019;76:505-14.

14. Yang K, Yao L, Li X, et al. A modified suture technique for transperitoneal laparoscopic dismembered pyeloplasty of pelviureteric junction obstruction. Urology 2015;85:263-7.

15. Clavien PA, Barkun J, de Oliveira ML, et al. The ClavienDindo classification of surgical complications: five-year experience. Ann Surg 2009;250:187-96.

16. Turk IA, Davis JW, Winkelmann B, et al. Laparoscopic dismembered pyeloplasty--the method of choice in the presence of an enlarged renal pelvis and crossing vessels. Eur Urol 2002;42:268-75.

17. Olweny EO, Park SK, Tan YK, et al. Perioperative comparison of robotic assisted laparoendoscopic single-site (LESS) pyeloplasty versus conventional LESS pyeloplasty. Eur Urol 2012;61:410-4.

18. Youssef RF, Spradling K, Yoon R, et al. Applications of three-dimensional printing technology in urological practice. BJU Int 2015;116:697-702.

19. Martelli N, Serrano C, van den Brink H, et al. Advantages and disadvantages of 3-dimensional printing in surgery: A systematic review. Surgery 2016;159:1485-500.

20. Zhang Y, Ge HW, Li NC, et al. Evaluation of threedimensional printing for laparoscopic partial nephrectomy of renal tumors: a preliminary report. World J Urol 2016;34:533-7.

21. Porpiglia F, Fiori C, Checcucci E, et al. Augmented
Reality Robot-assisted Radical Prostatectomy: Preliminary Experience. Urology 2018;115:184.

22. He Y, Luo S, Wu X, et al. Computed tomography angiography with $3 \mathrm{D}$ reconstruction in diagnosis of hydronephrosis cause by aberrant renal vessel: A case report and mini review. J Xray Sci Technol 2018;26:125-31.

23. Wang DS, Stolpen AH, Bird VG, et al. Correlation of preoperative three-dimensional magnetic resonance angiography with intraoperative findings in laparoscopic renal surgery. J Endourol 2005;19:193-9.

24. Cheung CL, Looi T, Lendvay TS, et al. Use of 3-dimensional printing technology and silicone modeling in surgical simulation: development and face validation in pediatric laparoscopic pyeloplasty. J Surg Educ 2014;71:762-7.

25. Bendre HH, Rajender A, Barbosa PV, et al. Robotic dismembered pyeloplasty surgical simulation using a 3D-printed silicone-based model: development, face validation and crowdsourced learning outcomes assessment. J Robot Surg 2020;14:897-902.

26. Thom MR, Haseebuddin M, Roytman TM, et al. Robotassisted pyeloplasty: outcomes for primary and secondary repairs, a single institution experience. Int Braz J Urol 2012;38:77-83.

27. Sivaraman A, Leveillee RJ, Patel MB, et al. Robot-assisted laparoscopic dismembered pyeloplasty for ureteropelvic junction obstruction: a multi-institutional experience. Urology 2012;79:351-5.

28. Atug F, Burgess SV, Castle EP, et al. Role of robotics in the management of secondary ureteropelvic junction obstruction. Int J Clin Pract 2006;60:9-11.

29. Fedelini P, Verze P, Meccariello C, et al. Intraoperative and postoperative complications of laparoscopic pyeloplasty: a single surgical team experience with 236 cases. J Endourol 2013;27:1224-9.

30. Lallas CD, Pak RW, Pagnani C, et al. The minimally invasive management of ureteropelvic junction obstruction in horseshoe kidneys. World J Urol 2011;29:91-5.

Cite this article as: Cheng $\mathrm{S}, \mathrm{Li} \mathrm{X}, \mathrm{Zhu} \mathrm{W}, \mathrm{Li} \mathrm{W}$, Wang J, Yang J, Wu J, Wang H, Zhang L, Li X, Zhou L. Real-time navigation by three-dimensional virtual reconstruction models in robot-assisted laparoscopic pyeloplasty for ureteropelvic junction obstruction: our initial experience. Transl Androl Urol 2021;10(1):125-133. doi: 10.21037/tau-20-1006 